\title{
Facilitating Quantitative Analysis of Atomic Scale 4D STEM Datasets
}

Zhen Chen ${ }^{1}$, Matthew Weyland ${ }^{2,3}$, Changxi Zheng ${ }^{1,4}$, Michael S. Fuhrer ${ }^{1}$, Adrian J. D’Alfonso ${ }^{5}$, Leslie J. Allen ${ }^{5}$, and Scott D. Findlay ${ }^{1}$

${ }^{1 .}$ School of Physics and Astronomy, Monash University, Clayton, Victoria 3800, Australia

2. Monash Centre for Electron Microscopy, Monash University, Clayton, Victoria 3800, Australia

3. Department of Materials Science and Engineering, Monash University, Clayton, Victoria 3800, Australia

4. Department of Civil Engineering, Monash University, Clayton 3800, Victoria, Australia

5. School of Physics, University of Melbourne, Parkville, Victoria 3010, Australia

Traditional scanning transmission electron microscopy (STEM) integrates the electron signal over a subset of scattering angles to form commonly used imaging modes. However, there is potentially much information contained in the full 2D convergent beam electron diffraction (CBED) patterns. The development of fast readout cameras has enabled 4D diffraction pattern (4D-STEM) datasets, i.e. 2D diffraction patterns at each probe position in a 2D scan, to be obtained with atomic scale electron probes [1, 2]. Synthesizing multiple imaging modes [1], differential phase contrast imaging [3] and ptychographic phase reconstruction [4] have been demonstrated using such 4D datasets. However, there are still many practical aspects to be investigated to facilitate quantitative analysis of such 4D datasets.

In this talk, we demonstrate quantitative analysis of 4D-STEM datasets. Using a fast direct detection camera, a Gatan K2-IS installed on a condenser lens aberration corrected FEI Titan electron microscope operated at $300 \mathrm{kV}$, we acquired 4D datasets from $\mathrm{SrTiO}_{3}$ and monolayer $\mathrm{MoS}_{2}$ specimens using an atomic scale convergent probe. One raw diffraction pattern and one diffraction pattern averaged from about 50 unit cells after scanning drift correction are shown in figures 1(a) and 1(b), respectively, for a $78 \AA$ thick $\mathrm{SrTiO}_{3}$ specimen and with a probe-forming aperture semi-angle, $\alpha$, of $21.3 \mathrm{mrad}$. Significant signal-to-noise enhancement is evident in the sharp edge of diffraction disk in figure 1(b). Figure 1(c) shows one example of a 4D dataset from $\mathrm{SrTiO}_{3}$ after repeat unit cell averaging.

Multiple imaging modes, such as bright field, annular bright field and annular dark field images can be synthesized from post-acquisition analyses on these large datasets. Figure 2(a) shows a coherent bright field image (collection angle $4.3 \mathrm{mrad}, \alpha=17.1 \mathrm{mrad}$ ) synthesized from a $4 \mathrm{D}$ dataset from $\mathrm{MoS}_{2}$. Absolute contrast scale comparison with simulations using the $\mu$ STEM software [5] showed good agreement. Such a comparison is shown in figure 2(b) for the coherent bright field image after repeat unit cell averaging. Quantitative differential phase contrast (DPC) imaging is also demonstrated. DPC phase reconstruction [6,7] is applied to the experimental datasets. Figure 2(c) shows the phase of the transmission function reconstructed from the raw DPC images of $\mathrm{MoS}_{2}$, while figure 2(d) shows the quantitative comparison between experiment and simulation after the repeat unit cell averaging. The Mo and S atoms can be seen more clearly in these reconstructed phases than in the STEM images. Methods to handle, reduce and analyze these huge datasets (hundreds of GBs) will also be discussed [8].

References:

[1] K Kimoto and K Ishizuka, Ultramicroscopy 111 (2011), p. 1111-1116.

[2] C Ophus et al, Microscopy and Microanalysis 20 (2014), p. 62-63. 
[3] K Müller et al, Nature Communications 5 (2014), p. 5653.

[4] TJ Pennycook et al, Ultramicroscopy 151 (2015), p. 160-167.

[5] LJ Allen et al, Ultramicroscopy 151 (2015), p. 11-22.

[6] EM Waddell and JN Chapman, Optik 54 (1979) p. 83-96.

[7] R Close et al, Ultramicroscopy 159 (2015), p. 124-137.

[8] The authors thank Drs. Peter Ercius and Jim Ciston for the assistance during the experiments. This work was supported by the Australian Research Council (Projects DP110102228, DP140102538 and DE130100739) and the Monash Centre for Electron Microscopy. Work at the Molecular Foundry was supported by the Office of Science, Office of Basic Energy Sciences, and Office of the U.S. Department of Energy under Contract No. DE-AC02-05CH11231.

(a)

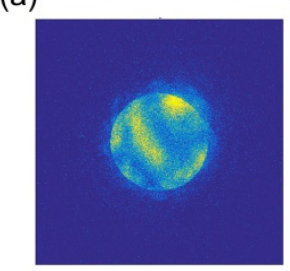

(b)

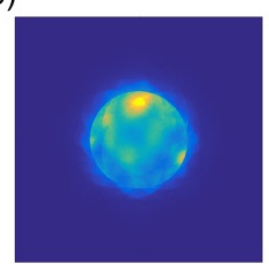

(c)

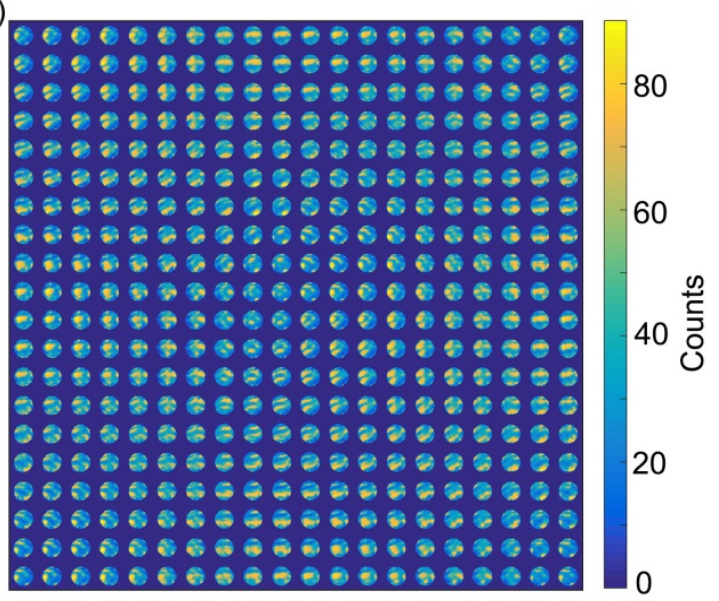

Figure 1. Convergent beam electron diffraction (CBED) patterns from a $4 \mathrm{D}$ dataset from a $\mathrm{SrTiO}_{3}$ specimen. (a) Raw CBED pattern, (b) averaged CBED pattern, and (c) a full 4D dataset after repeat unit cell averaging.

(a)

(b)
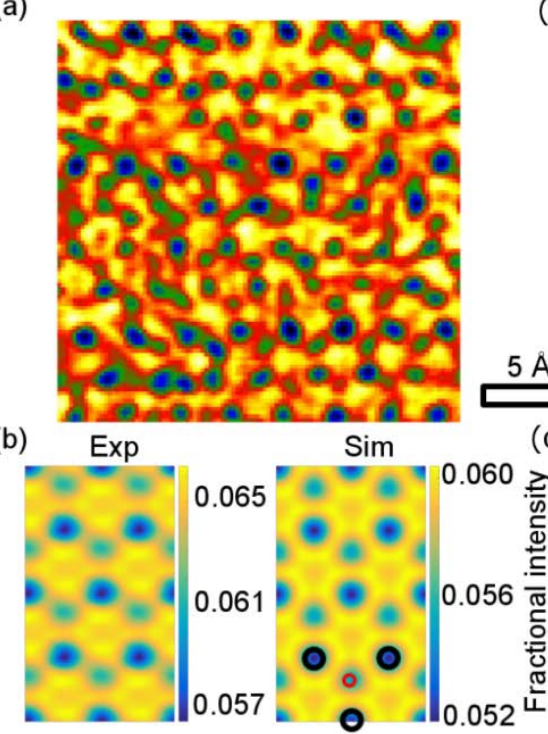

(c)

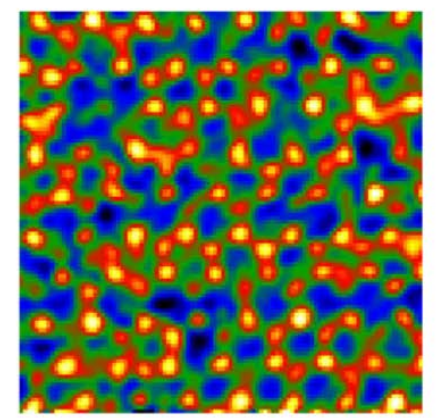

(d)

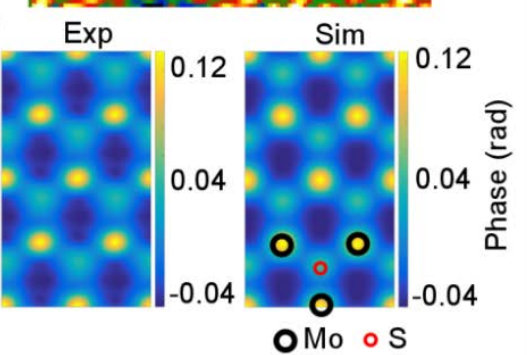

Figure 2. Images and transmission function phases from a monolayer $\mathrm{MoS}_{2}$ specimen. (a) Raw coherent bright field image (outer angle $\alpha / 4$ ). (b) Averaged experimental (left) and simulated (right) coherent bright field images. Phase reconstructions from the (c) raw and (d) repeat unit cell averaged DPC images. The scale bar pertains to the images in (a) and (c). 